\title{
Percepción de pacientes y enfermeras tratantes de Cuba sobre el autocuidado de mujeres mastectomizadas
}

\author{
Perception of patients and nursing staff from Cuba about self-care of \\ mastectomized women
}

Martín-Hernández Magny ${ }^{1}$, Torres-Esperón Julia Maricela², Mora Pérez Yuliet ${ }^{3}$,
Enriquez-González Carilaudy ${ }^{4},{ }^{*}$ Sánchez-Rodríguez José Rolando $^{5}$

${ }^{1}$ Doctora en Ciencias de la Enfermería. Profesora Auxiliar de Universidad de Ciencias Médicas. Santa Clara. Jefa de dpto de consulta externa, Hospital Universitario Oncológico Dr. Celestino Hernández Robau. Santa Clara. Cuba.

${ }^{2}$ Doctora en Ciencias de la salud. Profesor e investigador titular. Escuela Nacional de Salud Pública.

La Habana Cuba. e-mail: mtorresesperon7@gmail.com

${ }^{3}$ Doctora en Enfermería. Profesor Auxiliar e Investigador Agregado. Vicedirectora de Enfermería.

Hospital Gustavo Aldereguía Lima. Cienfuegos. Cuba. e-mail: yuliett.mora@gal.sld.cu

${ }^{4}$ Doctora en Enfermería. Profesor Auxiliar e Investigador Agregado. Universidad de Ciencias Médicas de Villa Clara. Cuba. e-mail: carilaudyeg@infomed.sld.cu

${ }^{5}$ Doctor en Enfermería. Profesor Asistente, Carrera de Enfermería. Universidad Arturo Prat. Chile.

*Autor para correspondencia: josersan@unap.cl

RECIBIDO: 10 de Octubre de 2020

APROBADO: 20 de Enero de 2021

DOI: $10.22370 /$ rev.mat.1.2021.2468

LOS AUTORES DECLARAN NO TENER CONFLICTO DE INTERESES

Palabras claves: Autocuidado, educación en enfermería, mastectomía, cáncer de mama, conductas relacionadas con la salud.

Key words: Self-care; nursing education; mastectomy; breast cancer; health-related behaviors.

\section{RESUMEN}

Introducción: El cáncer de mama es frecuente en mujeres. Su tratamiento puede llegar a ser muy invasivo, como es el caso de la mastectomía. Postcirugía pueden presentarse complicaciones relacionadas con la falta de orientaciones para el autocuidado, base de su recuperación.

Objetivo: Explorar desde la percepción de pacientes y enfermeras tratantes las necesidades del autocuidado de las mujeres mastectomizadas, para el diseño de una tecnología educativa.

Metodología: Estudio exploratorio fenomenológico, realizado en el Hospital Oncológico Dr. Celestino Hernández, Villa Clara, Cuba, durante 2018. La recolección de datos fue por entrevista grupal focalizada en diez pacientes mastectomizadas y doce enfermeras del servicio de cirugía. Las participantes se seleccionaron mediante muestreo de tipo homogéneo y bajo la condición común de que se tratara de pacientes mastectomizadas. Y en el caso de las enfermeras, la característica era que fueran del ser- vicio de cirugía, con cinco o más años de experiencia laboral. Se cauteló el rigor ético al cumplir con consentimiento informado, consideraciones éticas de la Asociación Médica Mundial y la Declaración de Helsinki.

Resultados: De las pacientes surge la categoría "necesidad de información para su autocuidado", con dos subcategorías: material impreso con información e informaciones necesarias para autocuidado de cirugía de mama. De las enfermeras emergió la categoría "preparación del personal de enfermería para orientar sobre el autocuidado de mujeres mastectomizadas".

Conclusiones: El estudio identificó la necesidad de contar con una tecnología educativa para promover el autocuidado de pacientes mastectomizadas, que fuera de fácil acceso y comprensible desde la construcción de las participantes. Además, debe contener sugerencias necesarias para prevenir complicaciones. Las enfermeras perciben que su preparación científica es esencial para entregar orientaciones sobre autocuidado. 


\section{ABSTRACT}

Introduction: Breast cancer is common in women and its treatment can be very invasive, such as mastectomy. Postoperative complications may occur related to the lack of guidelines for self-care.

Objective: explore from the perception of patients and treating nurses, the self-care needs of mastectomized patients for the design of an educational technology.

Methodology: exploratory study of phenomenological perspective. It was carried out at the Dr. Celestino Hernández Robau Oncological Hospital, Villa Clara, Cuba in 2018. The data collection was by focused group interview with ten mastectomized patients and twelve nurses from the surgery service. The participants were selected by homogeneous sampling. The characteristic of patient choice was having received a mastectomy and that of nurses who were from the surgery service, with more than five years of work experience. The ethical considerations of the World Medical Association and Declaration of Helsinki were met.

Results: From the patients the category arises: need for information for self-care, with two subcategories: printed material with information and information necessary for self-care for breast surgery. From the nurses, the category emerged: preparation of the nursing staff to guide on the self-care of women with mathematics.

Conclusions: The study identified the need for educational technology for the self-care of mastectomized patients, which was easily accessible, understandable from the construction of the participants, as well as containing the necessary suggestions to prevent complications. Nurses perceive that their scientific preparation is essential to provide guidance on self-care.

\section{INTRODUCCIÓN}

La Organización Mundial de la Salud (OMS) promueve el desarrollo de programas nacionales de lucha contra el cáncer de mama, integrándolo en prevención y vigilancia de las enfermedades no transmisibles. Esta institución reporta que en el mundo este tipo de cáncer ocupa el primer lugar de muerte por neoplasia maligna y que su impacto global se ha incrementado en dos veces (duplicado) en los últimos treinta años. (1)
En muchos países se han organizado políticas y estrategias para la prevención del cáncer mamario, por ser éste un problema de salud pública que afecta fundamentalmente a mujeres. En el caso de Cuba, se ha desarrollado un programa nacional para el control preventivo, manejo y tratamiento del cáncer de mama, que enfatiza en la educación y promoción de acciones de salud para mejorar conocimientos, actitudes y prácticas saludables en la población, así como, también, la detección precoz y la prevención de factores de riesgo a nivel poblacional, desde el modelo de salud familiar en Atención Primaria de Salud. (2)

Al cierre de 2018, las estadísticas de Cuba situaban dentro de las diez primeras causas de muerte las enfermedades del corazón, con una tasa de 228.2 por cien mil habitantes, seguida de las muertes por tumores malignos, con una tasa de 221.3. Entre las mujeres, la tasa de mortalidad más elevada corresponde al tumor maligno de tráquea, bronquios y pulmón. Le sigue a estos el de mama, con tasas superiores a nueve por cada cien mil habitantes. En la mortalidad de tumores malignos según localización de cáncer, el de mama ocupó el cuarto lugar, con 1.592 defunciones. De éstas sólo ocho fueron del sexo masculino. (3)

En Villa Clara, zona central de Cuba, las estadísticas de morbilidad y mortalidad por cáncer de mama también son elevadas en número de casos. Las defunciones en 2018, por tumores malignos de la mama, llegaron a 1.630, constituyéndose en la principal causa de muerte en el rango etario de 50 a 64 años. (3)

Esta afección ocasiona disímiles trastornos, tanto físicos y psicológicos como sociales. Ejemplo de ello es el estudio sobre significados de las actividades laborales para mujeres jóvenes con neoplasias de mama, en el que se obtuvo que "este cáncer y su tratamiento ocasionan sentimientos de las mujeres jóvenes acerca de tener que dejar de trabajar debido al cáncer de mama y su terapia; dejar el trabajo significaba molestia y desánimo; el cambio en los ingresos económicos generó preocupación con la contribución financiera, además de las trasformaciones físicas que cambian las actividades laborales, lo que significa limitación y discapacidad.(4) 
En el Hospital Universitario Oncológico Dr. Celestino Hernández Robau de Villa Clara, se atiende a mujeres con cáncer de mama de la región central de Cuba, las que reciben variados tratamientos: cirugía, quimioterapia, radioterapia, hormonoterapia o inmunoterapia. En la cirugía de mama unas de las técnicas quirúrgicas más empleada es la mastectomía combinada o no con otros tratamientos.

Tras la mastectomía, estas pacientes requieren atención individualizada del personal de enfermería, el que debe brindar los cuidados necesarios y ofrecer orientaciones para el fomento del autocuidado, como acciones deliberadas, intencionadas para la regulación del propio funcionamiento y desarrollo humano; práctica de actividades que llevan a cabo por su propia parte para mantener un funcionamiento sano y continuar con su desarrollo personal.(5)

El autocuidado en pacientes mastectomizadas contribuye a evitar complicaciones postquirúrgicas para una mejor evolución. Sin embargo, complicaciones como el linfedema, cuya prevención requiere de acciones de autocuidado, muchas veces no se pronostica desde el paciente y familia. (6) El modo como la persona se ve y se comporta frente a la necesidad de cuidados con el brazo afectado puede, muchas veces, revelar malestar emocional y físico que acarrea alteraciones en la calidad de vida. Para promover una atención que favorezca el bienestar físico y mental de esa persona, la profesional de enfermería puede valerse de cuidados que eleven la autoestima de la mujer. (7)

Para los investigadores brasileiros Pinto Coelho y colaboradores, le corresponde a la enfermera comprender la percepción de la mujer sobre sí misma, en relación con su imagen corporal para repensar su práctica profesional y buscar estrategias que aumenten la autoestima y mejoren la calidad de vida.(8) También procurar estrategias dentro de la función de cuidado profesional para prevenir complicaciones, postcirugía.

De los estudios encontrados por las autoras en una revisión integradora (9) se constató que en siete de ellos se abordaron guías de autocuidado para mujeres mastectomizadas, de los cuales dos utilizaron estudios descriptivos con cuestionarios y observación para identificar las necesidades de autocuidado de las pacientes. (10-11) No obstante, identificar es- tas necesidades desde la percepción de las personas que han vivido la experiencia y de los profesionales que las cuidan puede ser una alternativa loable para diseñar intervenciones para el autocuidado, como fue el caso del estudio que le da salida a este artículo.

De acuerdo con los registros estadísticos del hospital estudiado en 2017, de cincuenta pacientes operadas de mama en un mes, aproximadamente el 40\% de ellas acudía en busca de orientación por complicaciones, como seromas, linfedema, alteraciones de movilidad o sensibilidad de la zona operada. Dichas complicaciones están relacionadas de manera general con el autocuidado postcirugía, por lo que conocer la percepción de pacientes y enfermeras en relación con las necesidades de autocuidado y las vías para su orientación resulta importante para el diseño de intervenciones de enfermería y tecnologías educativas contextualizadas, en esta dirección.

Por consiguiente, el objetivo de este artículo es explorar desde la percepción de pacientes y enfermeras tratantes las necesidades de autocuidado de las mastectomizadas para el diseño de una tecnología educativa.

\section{METODOLOGÍA}

Estudio cualitativo exploratorio de perspectiva fenomenológica. La temporalidad de dicho estudio fue el año 2018, en el Hospital Oncológico Universitario Dr. Celestino Hernández, de la Provincia Villa Clara, Cuba.

Los datos fueron recolectados mediante la técnica de entrevista grupal focalizada, a dos grupos separados. Un grupo fue conformado por diez pacientes mastectomizadas y el otro por doce enfermeras tratantes vinculadas al servicio de cirugía oncológica. La selección de las participantes se realizó mediante muestreo no probabilístico de tipo homogéneo, en el que la característica común de elección para las mujeres fue haber recibido mastectomía y para las profesionales de enfermería que se desempeñaran en el servicio de cirugía oncológica, con más cinco de años de experiencia laboral. En cada grupo de participantes la entrevista grupal focalizada se realizó por separado. 
Se seleccionó esta técnica porque las entrevistas grupales constituyen el resultado de un esfuerzo colaborativo entre los entrevistados y el moderadorinvestigador, en el que el contenido del encuentro es el producto de un relato co-construido, o un texto negociado de manera contextual. (13) De esta manera, se capturaron los universos de significados, valores y experiencias de los participantes en relación con el objeto de estudio.

Las preguntas detonadoras para las pacientes mastectomizadas fueron dos: Previo a su cirugía, ¿Le hubiese gustado tener orientaciones de alguna manera en especial? ¿Cuáles?

Y la segunda fue ¿Cuáles conocimientos percibe usted, son necesarios para cuidarse mejor, post cirugía?

Para las enfermeras, en tanto, se usó una sola interrogante: En su experiencia de cuidado en pacientes con mastectomía, ¿qué intervenciones consideran ustedes, mejorarían el autocuidado efectivo post cirugía?

Cada sesión con grupo de participantes se organizó en base a las fases de desarrollo de las técnicas grupales: planteamiento de objetivos, preparación, organización y desarrollo del tema y análisis de la información. Se seleccionó un local con condiciones de espacio, ventilación e iluminación, se dispusieron los asientos en forma de herradura para visibilidad e intercambio entre participantes y la moderadora. Colaboraron tres auxiliares de investigación, licenciadas en enfermerías, con experiencia en la realización de estudios cualitativos y desarrollo de la metodología; una como observadora que realizó la relatoría de forma manual y las otras dos grabaron las sesiones y auxiliaron en los aspectos logísticos.

La moderadora en cada grupo de entrevista grupal focalizada explicó los objetivos, la importancia de la información a recolectar, las razones de la selección de los participantes y la dinámica de trabajo que se utilizaría durante la sesión. En la fase central de cada grupo se dirigió la conversación hacia la temática esencial, a partir de la introducción del tema seleccionado. El debate transcurrió de manera armónica, sobre la base del respeto y con participación de todas las participantes. Las sesiones tuvieron una duración aproximada de una hora y 30 minutos.
Con el fin de preservar el anonimato de los participantes y tamizar la información de cada grupo, se identificaron con letra "M" a las pacientes mastectomizadas y con letra "E" a las enfermeras. Todos los casos fueron enumerados de acuerdo con el orden de la palabra. Las narrativas fueron grabadas, transcritas y agrupadas en un cuerpo textual para realizar el análisis de contenido, que fue de tipo temáticocategorial, según Bardin,(14) donde emergieron de manera inductiva las categorías y subcategorías, lo cual posibilitó un proceso de horizontalización y descripción amplia del objeto en estudio.

La fiabilidad y validez del estudio se orientó en codificar dos veces cada entrevista grupal focalizada para buscar coincidencias de códigos y profundizar respuestas. Las percepciones y dificultades de interpretación de discursos se verificaron por triangulación con equipo de investigación y expertos externos del tema, hasta alcanzar acuerdos en asignación de categorías y sub-categorías, las cuales tuvieron características de exhaustividad, exclusión mutua, homogeneidad, pertinencia y fidelidad al tratar de hacer aportaciones con sustento al constructo teórico. Además, los investigadores aplicaron el principio de la reflexividad, como estrategia metodológica para asegurar calidad de la investigación en términos de credibilidad y rigurosidad metodológica.

El estudio tuvo en cuenta un control de sesgos en la investigación. Para evitar los asociados al observador o investigador y las técnicas de recolección y análisis de datos, se seleccionaron facilitadores con experiencia en la aplicación de la metodología a seguir, se tuvieron en cuenta diferentes alternativas para el registro de información de forma manual, a través de la grabación de las voces de los participantes. Para garantizar la veracidad de la información expresada, se garantizó asimismo un ambiente confortable, de confianza y respeto, con el establecimiento de normas de convivencia en el grupo y se explicó que toda la información recabada solo iba a ser usada con fines investigativos y con estricta privacidad.

El proyecto del estudio fue evaluado por el consejo científico del hospital de referencia y aprobado mediante el acuerdo $\mathrm{N}^{\circ} 48$ del 09 de junio de 2017 y presentado al Comité de ética para investigaciones del Hospital, el 15 de julio de ese mismo año, donde 
se obtuvo su aprobación para la ejecución. Posteriormente, se realiza una recolección de datos, previo a la firma del consentimiento informado por parte de las pacientes y profesionales de enfermería, que registró requisitos éticos de Ezekiel Emanuel. Se cumplieron normas y pautas de la Asociación Médica Mundial, así como, también, se cumplió con las consideraciones éticas establecidas según la Declaración de Helsinki en Fortaleza, Brasil, en 2013, para la realización de la investigación. (15) Se devolvieron los resultados de la investigación mediante informes técnicos a la dirección del hospital y el producto concluido a las pacientes y personal de enfermería participantes en el estudio.

\section{RESULTADOS}

Los temas que emergieron en la construcción de los sentidos y los significados de las afirmaciones se agruparon en categorías comunes, toda vez que se llevaron a cabo las etapas señaladas para el análisis fenomenológico.

Los resultados develaron una categoría desde las participantes, pacientes: "necesidad de información de cómo autocuidarse post cirugía" y dentro de ella dos subcategorías: "material impreso con información" e "informaciones necesarias para el autocuidado de cirugía de mama". En el caso de percepciones de los profesionales de enfermería, se obtuvo una categoría: "preparación del personal de enfermería para orientación sobre autocuidado".

La descripción más detallada de cada una se comenta a continuación.

Categoría: "necesidad de información de cómo autocuidarse post cirugía"

Subcategoría: "material impreso con información"

Los mayores significados y experiencias de las participantes que se recogen como resultados se enfocaron en la solicitud, necesidad y preocupación de un material impreso y de fácil manejo, que garantice la optimización y comprensión de su uso. Así se declaran en voces de las participantes, como se puede leer a seguir.

Pacientes: "...un material que me pueda llevar a mi casa (M2, M4), ...un librito con información (M3, M4), ...algo que nos guie como cuidarnos (M1, M5, M6)".
Profesionales de enfermería: ...algún folleto para entregar en la consulta (E1, E4, E7), ...un material impreso que explique cómo deben cuidarse (E5, E8, E10), ...deberíamos tener un material educativo para entregar a las mujeres operadas (E4, E12), ...si una guía que podría incluso entregarse en la consulta antes de la cirugía (E2, E7)".

Subcategoría: "informaciones necesarias para el autocuidado de cirugía de mama".

Tanto, las pacientes como las profesionales de enfermería delinearon los aspectos que deben estar incluidos en el material para el autocuidado de pacientes mastectomizadas, mediante la entrevista grupal focalizada. Las voces que respaldan la subcategoría así lo representan.

Pacientes: “..información sobre qué es el cáncer de mama y la mastectomía (M3, M5), ...actividades que podemos realizar (M3, M5, M7), ... cuidados a tener con ese brazo (M2, M8), ...información sobre trabajo que podemos hacer (M1, M4), ...si puedo tener hijos (M10), ...qué ropa puedo utilizar (M3, M5, M10), ... cuál es la mejor alimentación (M2, M5)), ...saber que deportes se puedo hacer (M8, M10), ...iy qué me dices de la recreación? (M4), ...los aspectos psicológicos son muy importantes (M3), ...imagen corporal interna y externa (M6, M9,) ...que opciones tenemos para prótesis, dónde y cómo obtener esa información (M4, M9)".

Profesionales de enfermería: “...cuidados en base a la evidencia, durante la hospitalización (E2, E5, E6), ...cuidados a tener con ese brazo (E5, E8, E11), ...los aspectos psicológicos son muy importantes (E4, E9, E11), ...imagen corporal interna y externa (E10, E11)".

Categoría: "preparación del personal de enfermería para orientar el autocuidado".

Los profesionales de enfermería reconocen la necesidad de prepararse en el tema y aunque tengan algún material de apoyo; sus conocimientos sobre orientaciones de autocuidado en mujeres mastectomizadas deben ser profundos para cualquier duda que surja.

... saber cada día más sobre este tema, es una necesidad (E8, E10), ... ¿cómo vamos a orientar sin prepararnos? (E4), ...las preguntas que nos 
hacen las pacientes todos los días nos obligan a prepararnos (E3, E4, E9), ... deberíamos recibir más capacitación del tema (E6, E9, E10, E11) ... nosotras debemos buscar también información siempre (E1, E5, E7), ...somos fuentes de información confiable para sus dudas (E2)".

\section{DISCUSIÓN}

En la medida que en los discursos se identificó el posicionamiento de los participantes, como beneficiarios de sus propuestas, se retrotrajeron las experiencias que les permitieron descubrir los motivos de porqué y para qué de la acción de autocuidado, con miras a entregar sentido y significado a sus actuaciones, justificadas por la teoría social de Alfred Schutz,(16) que señala que en el mundo de la vida cotidiana se construyen los proyectos y los planes de vida.(17)

Las pacientes mastectomizadas desde el escenario social y dinámico donde se interrelacionan, llevan a cabo las acciones y los nexos con sujetos que comparten un universo cultural de significaciones intersubjetivas con comprensiones de sus vivencias y necesidades.(18)

La subcategoría: "material impreso con información" fue determinante para el estudio, dado que marcó la pauta para construir una tecnología educativa que puede servir de guía de autocuidado, ya que, de acuerdo con la revisión de la literatura científica, el formato más común utilizado para estos fines es el de las guías. Así se expone en los estudios realizados por Capllonch Teba, Santos Luna, Bañon Amat, Aranda de Lara y Figueroa Portillo, (19-23) cuyo objetivo estuvo centrado en elaboración de guías, protocolos y planes de atención para cuidados o autocuidados a pacientes mastectomizadas en formatos digitales, libro o manual a ser explicitados en consultas de enfermería o en el servicio hospitalario.

En la subcategoría: "informaciones necesarias para autocuidado post cirugía de mama", los aspectos psicológicos y la imagen corporal ocupan un lugar importante en los discursos, a través del uso del acopio de experiencias de las participantes, la cual Schutz(16) denomina "modo pragmático" para orientarse en la nueva praxis de su vida cotidiana. Éstas contribuyen a vivenciar el proceso desde la nueva experiencia de vida como mujer mastectomizada, con varios acervos de conocimientos que deberían desarrollar para su nuevo modo de vida con tipificaciones y abstracciones que explican los comportamientos pragmáticos de las participantes.

La literatura revela lo que significa para una mujer vivir con cáncer de mama y estar mastectomizada, al tener que redescubrir emociones, capacidades de adaptación, ser consecuente y responsables de su tratamiento y disociar mente y cuerpo. El redescubrir emociones compromete al proceso de afrontamiento frente a una mastectomía, que requiere un esfuerzo integral de la persona para superar esta situación de salud y que la hace vulnerable y dependiente emocionalmente, donde la mujer genera la necesidad de apoyo emocional para fortalecer la capacidad de resiliencia ante síntomas, signos y cambios asociados al período post cirugía, con una fuerte significación de vida, de los lazos familiares y los proceso y subjetividades respecto a la muerte. $(4,6,21)$ Por ello, un material de apoyo con informaciones necesarias para estas pacientes es vital tras la cirugía.

En la revisión sistemática realizada por Varela y colaboradores, con el objetivo de describir los instrumentos para evaluar la imagen corporal de mujeres con cáncer de mama en población latinoamericana, se evidencia que la insatisfacción de las mismas con el cuerpo, después de descubierto el cáncer de mama, es mayor cuando atraviesan algunas condiciones como el linfedema o el uso de prótesis después de la mastectomía y el aumento de peso. Se encontró que la vulnerabilidad psicológica es mayor cuando la mujer considera que su apariencia va definir su autoestima. (21)

Para estos autores, dados los estigmas y preconceptos, se entiende que los profesionales de la salud, en particular los de Enfermería, pueden ser preservadores en el enfrentamiento de los desafíos, para estimular el retorno a las actividades sociales y funcionales de mujeres con mastectomías, así como promover su autoestima y autoconfianza. Se conoce que personas más optimistas presentan mayor satisfacción con la imagen corporal y una mejor adaptación psicológica a la nueva condición de salud, lo que repercute en mejores tasas de sobrevida y calidad de vida. (24). 
Desde la perspectiva del referente teórico, la comprensión del fenómeno de las experiencias vividas en la combinación de relaciones intersubjetivas satisfactorias, que han definido las acciones ejecutoras en el proceso de rehabilitación y autocuidado de mujeres mastectomizadas devela la necesidad de conocimientos que le permitan una mejor recuperación. (17)

Otros estudios abordan programas con la finalidad de enseñar pasos, procedimientos rehabilitadores que potencien el autocuidado desde el período postquirúrgico inmediato y su seguimiento en el hogar para la vida, así como tener en cuenta la recuperación desde el primer momento. Para Schütz, (16) las tipificaciones se refieren más a la relación existente con las situaciones que se establecen y se preparan con y para las personas. En ese sentido, las pacientes mastectomizadas serían responsables de su autocuidado al aplicar el algoritmo de programaciones con tiempos, esfuerzos y técnicas aprendidas en su proceso de rehabilitación postquirúrgica.

Al respecto García Rosado refiere que "un programa de seguimiento en pacientes hospitalizados después de recibir alta médica hospitalaria puede ser considerado como herramienta o método óptimo para su seguimiento, al mejorar la atención y satisfacción general. La rehabilitación debe ser iniciada en el hospital, con asistencia especializada multidisciplinaria, enseñando el autocuidado... al paciente y su familia". (24).

Con puntos de coincidencia, los autores de esta investigación consideran que estos aspectos pueden ser valorados para la posterior implementación de una intervención para pacientes mastectomizadas.

Dicha recomendación también se reflejó en las síntesis del conocimiento de una revisión integradora realizada por Menis Sasakiy colaboradores, (25) en la que se afirman que "la rehabilitación de toda paciente mastectomizada debe ser iniciada en el centro asistencial, con enfoque multidisciplinario especializado, para el paciente y su familia, enseñándoles el autocuidado..., así como estrategias para el retorno laboral y social...". A este respecto Schütz,(27) señala que las personas, al encontrarse con un nuevo problema en su actuar cotidiano, objetivo o intersubjetivo, deberían buscar tipificaciones y procedimien- tos, para establecer otras nuevas formas de acción que permitieran respuestas más apropiadas al nuevo mundo desconocido.

Pinto Coelho \& colaboradores, a partir de un estudio fenomenológico sobre atención de enfermería en la perspectiva del mundo de las mujeres con linfedema en el tratamiento del cáncer de mama, consideran que "frente al fenómeno develado por las mujeres mastectomizadas, se entiende que el profesional de enfermería puede contribuir significativamente, tanto en la gestión de los cuidados con el brazo edematoso, como en utilización de estrategias de educación para la salud, que propicie mejor calidad de vida de las mujeres".(8)

Según Schütz, (27) la realidad del sentido común y vivencias se consideran culturalmente universales, sin embargo, su expresión en la vida individual depende de la experiencia construida por el sujeto en su realidad social. Como es el caso de los profesionales de enfermería, los que son responsables de ese cuidado humanizado que cobra gran controversia al momento de direccionar la mujer mastectomizada hacia toma de decisiones y participación clínica, respecto a la continuidad de cuidados y tratamiento de su enfermedad, sabiendo que muchas veces sus preferencias son difíciles de predecir, debido a que todo implica novedad y sufrimiento para la persona y su familia.

García Rosado precisa: "La enfermera como parte del equipo de salud es responsable directa, teniendo como función el nivel preventivo promocional y atendiendo las necesidades educativas que tiene el ser humano, es necesario investigar con el propósito de demostrar la efectividad de los programas educativos..."(24). Así se confirma en los estudios que evalúan la efectividad de intervenciones de enfermería para la mejora del autocuidado,(27-29) donde sus autores se pronuncian a favor del valor de mejorar la preparación del personal de enfermería para garantizar la aplicación y los mejores resultados.

Por ejemplo, Cruz Acha \& colbs, (27) en su estudio sobre la efectividad de un programa educativo para mejorar el autocuidado en pacientes con ostomía, manifiestan que "... el rol de la enfermera, consiste en ayudar al paciente a que logre conseguir el 
autocuidado utilizando cinco modos de asistencia: actuar, guiar, apoyar, procurar un entorno que favorezca el desarrollo de la persona y enseñar".

En este sentido los investigadores del presente estudio reflexionan que los cuidados requeridos durante el tratamiento, seguimiento y control de mujeres mastectomizadas se modifican de acuerdo con el itinerario propio de la enfermedad y en respuesta ante situaciones imprevistas, donde los profesionales de salud juegan un rol esencial para el sostén emocional y cuidados físicos durante la hospitalización, el proceso del tratamiento y la rehabilitación.

Por su parte, Almendárez Saavedra y colaboradores, en un estudio pre-experimental sobre prácticas de autocuidado de pacientes enterostomizados, afirman que "el nivel de conocimientos sobre prácticas de autocuidado se incrementó en los pacientes, después de la intervención educativa de enfermería". (28).

Para Culha \& colaboradores en una investigación sobre eficacia de la educación de autocuidado en pacientes con estomas "el individuo que recibe la suficiente educación sobre la enfermedad tendrá suficiente autocuidado, independencia y adaptación". (29).

En concordancia con revisión integradora realizada por Diego-Cordero \& colaboradores, las mujeres mastectomizadas abordan en sus vivencias los aspectos emocionales y sexuales, factores sociales y culturales y los mecanismos de adaptación al cáncer de mama en relación a su cultura.(30) Estos aspectos son esenciales para el cuidado post cirugía, para evitar incertidumbre y el miedo derivados de los significados sociales que se han construido entorno a una radical de mama, como un padecimiento que sitúa a la persona en proximidad con la muerte y porque no se sabe con certeza que va a pasar con la mujer post cirugía de su mama.

Como puede observarse, son varios los estudios que demuestran el valor y la efectividad de las consultas de enfermería, como parte de la educación sobre el autocuidado en estas pacientes, tanto para tratamientos quirúrgicos como para el control de enfermedades crónicas y su seguimiento. Se destacan también las consultas de consejería de enfermería.
Es por ello que, estos resultados constituyeron el insumo para una intervención de enfermería que responde a las tendencias actuales de la disciplina y ciencia de la enfermería.

Como los resultados expuestos en este artículo forman parte de un proyecto cuyo objetivo fundamental es el diseño de una intervención de enfermería para el autocuidado de mujeres mastectomizadas, se ofrece la posibilidad de identificar las necesidades de autocuidado desde los sujetos diana, aspecto que es cada vez más empleado para traducir el conocimiento científico al conocimiento popular.

\section{CONCLUSIONES}

La investigación proporcionó respuestas de significados de las experiencias vividas por mujeres mastectomizadas sobre la necesidad de una tecnología educativa para el autocuidado post cirugía, que fuera de fácil acceso, comprensible desde la construcción de los participantes y, además, capaz de contener recomendaciones necesarias para prevenir complicaciones. Desde los profesionales de enfermería se reconoce la importancia de iniciar indicaciones de autocuidado desde la hospitalización, bajo conocimientos sólidos y con apoyo de materiales que faciliten al paciente orientaciones comprensibles y efectivas para prevenir complicaciones. Dado que este estudio fue la primera tarea del proyecto en el que está inserto, sus resultados fueron pautas decisivas para el diseño de una intervención de enfermería en pacientes mastectomizadas.

\section{LIMITACIONES}

En Cuba, en el área de enfermería, existen escasos estudios publicados con relación al tema. Se reconoce inclusión en esta investigación de artículos originales, pudiendo existir sesgo de publicación por información científica en bases de datos de congresos y simposios que no pudieron ser incluidas al no tener acceso.

Otra restricción es la ausencia de artículos originales de diseños longitudinales prospectivos, que describan seguimientos de cohortes de pacientes mastectomizadas para evaluar su evolución post intervención de cuidados integrales y con tecnología educativa. 


\section{REFERENCIAS}

1. Organización Mundial de la Salud. Cáncer, Nota descriptiva Febrero de 2017 [Internet]. Ginebra: OMS; 2017. [citado 26 Abr 2017]. Disponible en: http://www.who.int/mediacentre/factsheets / fs297/es/

2. Centro Nacional de Información de Ciencias Médicas. Cáncer de mama. Prevención. Bibliomed. [Internet]. 2016. [citado 23 Abr 2019]; 23(5): [aprox. 2 p.]. Disponible en: https://files.sld.cu/bmn/files/2016/05/bibliomed-mayo-2016.pdf

3. Ministerio de Salud Pública de Cuba. Dirección de Registros Médicos y Estadísticas de Salud. Anuario Estadístico de Salud. [internet]. 2018. La Habana, 08 de abr, 2019. [citado 14 May 2020]. 101-06. Disponible en: https://files.sld.cu/bvscuba/files/2019/04/Anuario-Electr\%C3\%B3nicoEspa\%C3\%B1ol-2018-ed-2019-compressed.pdf

4. Magalhães PAP, Loyola EAC, Dupas G, Borges ML, Paterra TSV, Panobianco MS. O significado das atividades laboráis para mulheres jovens com neoplasias da mama. Texto Contexto Enferm [Internet]. 2020. [12 Jun 2020];29:[aprox. 3 p.]. Disponible en: https://www.scielo.br/pdf/tce/v29/pt_1980265X-tce-29-e20180422.pdf

5. MarrinerTomey A, RaileAlligood M. Modelos y teorías en enfermería [Internet]. 6 ed. Barcelona: ElsevierMosby; 2015 [citado 23 Abr 2019]. Disponible en: https://books.google.es/books?id=FLEszO8XGT $\mathrm{UC} \&$ printsec $=$ frontcover\&hl $=\mathrm{es} \# \mathrm{v}=$ onepage $\& \mathrm{q} \& \mathrm{f}=\mathrm{fa}$ lse

6. Almeida TG, Comassetto I, Alves KMC, Santos AAP, Silva JMO, Trezza MCSF. Vivência da mulher jovem com câncer de mama e mastectomizada. Esc Anna Nery. [online]. 2015.19(3):432-8. Disponible en: http://dx.doi.org/10.5935/1414- 8145.20150057

7. Gomes NS, Soares MBO, Silva SR. Autoestima e qualidade de vida de mulheres submetidas à cirurgia oncológica de mama. Rev Min Enferm. [Internet]. 2015 [citado 4 Mar2020];19(2):[aprox. 3 p.]. Disponible en: https://www.dx.doi.org/10.5935/14152762.20150030

8. Do Carmo Pinto Coelho PA, Arantes EE, de Oliveira IE, Moreira Chagas M, Simões Cardoso de MC, Vasconselos AT. Cuidado de enfermagem na perspectiva do mundo da vida da mulher que vivencia linfedema de corrente do tratamento de câncer de mama. Esc Anna Nery. [Internet]. 2020. [cited 26 Mar 2020]; 24 (2):[aprox. 5 p.]. Disponible en: https://www.scielo.br/scielo.php?script=sci_ arttext\&pid=S1414-81452020000200206\&lng=
9. Martín Hernández M, Pérez Mora Y, Torres Esperón J. Guías de autocuidado en las pacientes con cáncer de mamas mastectomizadas. Medicentro Electrónica. [internet]. (2020). [citado 20dic 2020]; 24(4):805-818. Disponible en: http://www. medicentro.sld.cu/index.php/medicentro/article/ view/3232

10. Cuamatzi Peña, MT. Gómez Pérez, J. Manual de autocuidado del puerperio, alteraciones gineco-obstétricas y rehabilitación de la mastectomía. UNAM, FES Zaragoza, mayo de 2015. p. 99. Disponible en: https://procesoreproductivofeszaragoza. files.wordpress.com/2017/11/2c2b0-manual-deautocuidado-del-puerperio1.pdf

11. Aranda de Lara, A. Ballesteros León, R. Cuadrado Muñoz, MA. Guía de cuidados para mujeres mastectomizadas. Edición: Dirección de Enfermería. Unidad de Calidad, Docencia e Investigación de Enfermería. División de Enfermería. HOSPITAL UNIVERSITARIO REINA SOFÍA. Ediciones Gráficas Vistalegre. Disponible en: http://www.sld.cu/galerias/ pdf/sitios/rehabilitacion/mastectomia.pdf

12. Freidin B. Revisando el uso de grupos focalizados en la investigación social. Rev Latinoam Metodol Cienc Soc. [Internet]. 2016. [citado18 Jul 2018]; 6(1): [aprox. 4 p]. Disponible en: https://sedici.unlp.edu. ar/bitstream/handle/10915/54509/Documento_ completo.pdf-PDFA.pdf? sequence $=1 \&$ isAllowed $=y$

13. Bardin L. Análise de conteúdo. Edición 70. Brasil. Editorial: Casa de Ideias.[Internet]. 2016. Disponible en: https://madmunifacs.files.wordpress. com/2016/08/anc3allise-de-contec3bado-laurence-bardin.pdf

14. World Medical Association. Declaración de Helsinki, Fortaleza, Brasil, Paris: Asociación Médica mundial [Internet]. Finlandia: WMA; 2013 [citado 23 Abr 2019]. Disponible en: https://www. uchile.cl/documentos/declaracion-de-helsinki2013pdf_111779_0_3900.pdf

15. Guerrero Castañeda R.F, González Soto C.E, Jiménez González M.J. La salud y su cuidado como relación cultural intersubjetiva: reflexión fenomenológica en Schütz. Enfermería Actual de Costa Rica. [Internet]. 2020. [cited 2020 Oct 10]; (39): 23644. Disponible en: http://dx.doi.org/10.15517/revenf. v0i39.40680

16. Moreno González MM, Galarza Tejada DM, Tejada Tayabas LM. Experiencias del cuidado familiar durante el cáncer de mama: la perspectiva de los cuidadores. Rev. esc. enferm. USP. [Internet]. 2019 [cited 2020 June 30] ; 53: e03466. Disponible en: https://doi.org/10.1590/s1980-220x2018012203466 
17. Capllonch Teba V. Cuidados de enfermería en pacientes con cáncer de mama sometidas a radioterapia. Inquietudes Rev Enferm [Internet]. 2015. [citado 31 mar 2020]; 20(49): [aprox. 3 p.]. Disponible en: https://dialnet.unirioja.es/servlet/ articulo? codigo $=5408067$

18. Santos Luna J, Quezada T. Propuesta de atención de enfermería en el cáncer de mama en el Hospital Oncológico Solca Machala Dr. Wilson Franco Cruz. [tesis]. Machala: Universidad Técnica de Machala; 2015. Disponible en: https://repositorio.utmachala.edu.ec/bitstream/48000/7277/1/ TESIS\%20CANCER\%20DE\%20MAMA.pdf

19. Bañón Amat I, Castejón Navarro J, Cervantes Berná A, Espinosa Cabrera MA, Gamayo Serna A, Gil Gil-Albaladejo F, et al. Guía de cuidados para mujeres mastectomizadas [Internet]. España: Hospital Vega Baja.[Internet]. 2008 [citado 14 May 2020]. Disponible en: https://www.dep21.san.gva.es/deporihuela/wp-content/uploads/2009/12/GUIA-MAMA.pdf

20. Aranda de Lara A, Ballesteros León R, Cuadrado Muñoz MA, González Carmona J, Goñi Orellana C, de Gracia Gutiérrez J. Guía de cuidados para mujeres mastectomizadas [Internet]. Córdoba: Hospital Universitario Reina Sofía; 2000 [citado 31 Mar 2020]. Disponible en: https://docplayer.es/1680226Guia-de-cuidados-para-mujeres-mastectomizadas. html

21. Figueroa Portillo SM. María Guamán ÁG. Guerrero Inga AF. Protocolo de autocuidado en pacientes postmastectomizadas que acuden a consulta externa de oncología clínica y quimioterapia del hospital "Vicente Corral Moscoso" [tesis]. Cuenca: Universidad de Cuenca; 2012. Disponible en: https://dspace.ucuenca.edu.ec/bitstream/123456789/3573/1/Tesis\%20de\%20Pregrado.pdf

22. Hermosilla Ávila A, Sanhueza Alvarado O. La vivencia de los pacientes con cáncer y el cuidado de enfermería. Rev Cuid. [Internet];2020. [citado 23 Abr 2019]; 11(1): e782. disponible en: https://dx.doi. org/10.15649/cuidarte.782

23. Varela MRF, Sierra MDV, Heredia MER, Delgado RM. Evaluación de la imagen corporal en mujeres con cáncer de mama: una revisión sistemática. Univ Psychol [Internet]. 2017. [citado 31 mar 2020];16(4): [aprox. 3 p.]. Disponible en: https://www.redalyc. org/jatsRepo/647/64753569009/64753569009.pdf
24. García Rosado MF. Efectividad del programa educativo "recordar es vivir" en el nivel de conocimiento sobre autocuidado de la memoria en adultos mayores. nuevo chimbote, 2014. [tesis]. Chimbote: Universidad Nacional del Santa; 2015. Disponible en: http://repositorio.uns.edu.pe/bitstream/handle/ UNS/1899/27201.pdf?sequence=1\&isAllowed=y

25. Menis Sasaki VD, da Silva Teles AA; Silva de Lima M, Costa JC, Braga Lisboa B, Megumi Sonobe H. Reabilitação de pessoas com estomia intestinal: revisão integrativa. Rev Enferm [Internet]. 2017. [citado 31 Mar 2020];11(Supl 4):[aprox. 4 p.] Disponible en: https://pdfs.semanticscholar.org/a4c6/2e891e2 2a700af9c078e774d21e73478b52b.pdf

26. Gros AE. Tipificaciones y acervo de conocimiento en la fenomenología social de Alfred Schutz: Una reconstrucción teórico-sistemática. Revista Mexicana de Ciencias Políticas y Sociales. [Internet]. 2017. [citado 27 Jul 2020]; 62(231): 23-45. disponible en: https://www.sciencedirect.com/science/article/pii/S0185191817300375

27. Cruz Acha LC, Pamo Tapia MC. Efectividad del programa educativo de autocuidado en pacientes con ostomía [tesis]. Lima: Universidad Privada Norbert Wiener; 2018. Disponible en: http://repositorio.uwiener.edu.pe/bitstream/ handle/123456789/2267/ESPECIALIDAD\%20 \%20 Milagros\%20 Cecilia\%20 Pamo\%20Tapia. pdf? sequence $=1 \&$ isAllowed $=y$

28. Almendárez Saavedra JA, Landeros López M, Hernández Castañón MA, Galarza Maya Y, Guerrero Hernández $M$. Prácticas de autocuidado de pacientes enterostomizados antes y después de intervención educativa de enfermería. Rev Enferm Inst Mex Seg Soc. [Internet]. 2015. [citado 20 Nov 2019]; 23(2): [aprox. 3 p.]. Disponible en: http://revistaenfermeria.imss.gob.mx/editorial/index.php/revista enfermería/article/view/48/74

29. Culha I, Kosgeroglu N, Bolluk O. Eficacia de la educación de autocuidado en pacientes con estomas. J Nurs Health Sci [Internet]. 2016 [citado 20 Nov 2019];5(5):[aprox. 3 p.]. Disponible en: www.iosrjournals.org/iosr-jnhs/papers/vol5-issue2/Version-1/ J05217076.pdf

30. Diego-Cordero, R., Fernández-Fernández, $\mathrm{M}^{\mathrm{a}}$., \& Vega-Escaño, J. Cultura y espiritualidad en la vivencia del cáncer de mama y la mastectomía. Cultura de los Cuidados (Edición digital). [internet]. (2020).[citado 15jun 2020]; 24(57):9-26.Disponible en: http://dx.doi.org/10.14198/cuid.2020.57.02 